\title{
1 Predicting residential building age from map data
}

2 Rosser, J.F. ${ }^{* 1}$, Boyd, D.S. ${ }^{1}$, Long, G. $^{1}$, Zakhary, S. ${ }^{1}$, Mao,,${ }^{1}$, , Robinson, D. ${ }^{2}$

$3 \quad{ }^{1}$ University of Nottingham, Nottingham, UK

$4 \quad{ }^{2}$ University of Sheffield, Sheffield, UK.

5

$6 *$ Corresponding author

7 Julian.Rosser@ nottingham.ac.uk

8 University of Nottingham

9 Nottingham

$10 \quad$ NG7 2RD

11

12 Funding acknowledgements:

13 This work was supported by the Leverhulme Trust (grant number RP 2013-SL-015). Additional

14 funding was provided from Ordnance Survey and 1Spatial Ltd.

15

16 Conflicts of interest:

17 The author(s) declared no potential conflicts of interest with respect to the research, authorship, 18 and/or publication of this article.

19

20 


\section{Predicting residential building age from map data}

The age of a building influences its form and fabric composition and this in turn is critical to inferring its energy performance. However, often this data is unknown. In this paper, we present a methodology to automatically identify the construction period of houses, for the purpose of urban energy modelling and simulation. We describe two major stages to achieving this - a per-building classification model and post-classification analysis to improve the accuracy of the class inferences. In the first stage, we extract measures of the morphology and neighbourhood characteristics from readily available topographic mapping, a high-resolution Digital Surface Model and statistical boundary data. These measures are then used as features within a random forest classifier to infer an age category for each building. We evaluate various predictive model combinations based on scenarios of available data, evaluating these using 5-fold cross-validation to train and tune the classifier hyper-parameters based on a sample of city properties. A separate sample estimated the best performing crossvalidated model as achieving $77 \%$ accuracy. In the second stage, we improve the inferred per-building age classification (for a spatially contiguous neighbourhood test sample) through aggregating prediction probabilities using different methods of spatial reasoning. We report on three methods for achieving this based on adjacency relations, near neighbour graph analysis and graph-cuts label optimisation. We show that post-processing can improve the accuracy by up to 8 percentage points.

Keywords: building age; energy modelling; prediction; classification optimisation; MasterMap; Digital Surface Model;

\section{Introduction}

Buildings are responsible for $32 \%$ of global energy usage and $19 \%$ of energy-related greenhouse gas emissions (Lucon et al. 2014). Residential building stocks can be a major factor in determining this demand. In the UK, for example, domestic buildings are estimated to be responsible for $29 \%$ of national energy use (BEIS 2017). Reducing the energy demand imposed by domestic buildings requires accurate modelling of the current energy use of the housing stock and precise targeting of effective retrofit 
1 measures (Shorrock and Dunster 1997, Shorrock et al. 2005, Davies and Oreszczyn

2 2012). One important aspect in this modelling of a residential building's energy

3 demands is the capture of the time period in which it was constructed (i.e., its age or

4 construction period). This importance is, largely, due to the fact that this period

5 determines the morphology, façade design and the materials used, which impact on a

6 building's energy performance when used for habitation.

7 Several studies have shown how the age or construction period of buildings is a key

8 factor in modelling their energy usage (Firth et al. 2010, Tooke et al. 2014, Aksoezen et

9 al. 2015, Nouvel et al. 2017). In particular, it can be used to determine the building

10 regulations (if applicable) in place at the time of construction and provide a powerful

11 proxy for estimating a range of attributes that greatly affect its energy performance,

12 such as:

13 - the fabric of the built envelope (wall and roof types and associated the

14 construction methods),

15 - glazing types and ratios,

16 - potential energy retrofit measures that could be implemented and the degree of

17 disruption involved in their addition,

18 - storey heights.

19 The construction period is commonly used in conjunction with the built form of a

20 property (e.g. semi-detached, terraced, detached) as the basis for creating housing

21 archetypes that are used in housing stock energy modelling (such as Mavrogianni et al.

22 (2012), Davila et al. (2016), Firth et al. (2010) and Sousa et al. (2017)).

23 Identifying when a building was constructed also has a variety of important

24 applications outside of energy modelling. In the context of natural hazards, for example,

25 building age is used to help in assessing the vulnerability of properties to earthquakes 
1 (Steimen et al. 2004, Wieland et al. 2012). Similarly, the vulnerability of buildings to

2 damage by flood events is linked to the property age, which acts as an indicator of the

3 type of foundation, wall and roof construction (Fedeski and Gwilliam 2007). Models

4 estimating landslide risk also incorporate construction period (Uzielli et al. 2015).

5 In the UK and other countries, digital databases of construction period (and thus

6 building age) are not readily available. Furthermore, even when a dataset of building

7 age is available for an area, perhaps created through manual digitising of historic

8 mapping, it may not be suitable for assessing building energy use. For example, the data

9 may not be up-to-date, or the choice of age band is not appropriate or sufficiently

10 detailed for energy modelling purposes. Therefore, there is a need for a method that can

11 automatically identify, at the per-building level and according to a classification that is

12 meaningful for energy modelling, the construction period of properties that is applicable

13 at the urban, regional or national scale.

14 As urban areas develop over time, the construction and style of buildings

15 change. These changes can be apparent in the geometric shape and location of

16 buildings, and lead to particular spatial characteristics in the neighbourhoods of built-up

17 areas (Yu et al. 2010, Hermosilla et al. 2012). The geometry of the urban blocks can

18 indicate the type of buildings found there (Wurm et al. 2016), and also help in enabling

19 identification of areas corresponding to particular times in a city's development

20 (Barnsley and Barr 1997, Hermosilla et al. 2014). In this work, we exploit this

21 information to estimate a construction period of residential buildings. In particular, we

22 demonstrate a predictive modelling approach to infer an age automatically based on a

23 range of geometric and neighbourhood features, derived from commonly available

24 datasets. We treat the age of the building as an era, classified according to five periods

25 of urban construction. These include pre-1915, 1915 - 1944, 1945 - 1964, 1965 - 1979, 
1 and post-1980. These construction periods reflect those of the English Housing Survey

2 (EHS) (DCLG 2015) - a common source of statistical data describing the UK residential

3 building stock, which is used for energy modelling. For example, the EHS can provide

4 details on the physical construction characteristics linked to geographic regions

5 according to categories of age and form and has thus provided a basis for many housing

$6 \quad$ stock energy models (Sousa et al. 2017).

$7 \quad$ Our contributions include: 1) a detailed description and analysis of a statistical

8 method for inferring residential building construction period that is important for

9 modelling energy usage; 2) improved statistical inferences via post-classification

10 approaches based on spatial reasoning of the scene; 3) quantitative assessment of

11 different accuracy metrics obtainable for different sample sizes and model

12 combinations.

\section{Related work}

14 In this section, we review previous work on the identification of the age or

15 construction period of stocks of buildings. In this we focus our review on the

16 identification of per-building attributes, rather than the characterisation of urban

17 structure (i.e., Hermosilla et al. (2014)).

18 Meinel et al. (2009) describe a system which integrates spatial data from

19 mapping agencies with geo-referenced digital airborne imagery. The authors undertook

20 a process of cross-referencing of the geometric features of building footprints and the

21 neighbourhood structure against a large sample set building types and neighbourhood

22 forms, thus enabling the classification of building types. However, their construction

23 period focused on buildings newer than 1984 due to constraints on the available data.

$24 \quad$ Van Hoesen and Letendre (2013) analysed historical maps to determine building

25 age for identifying suitable properties for implementing energy efficiency measures. 
1 The authors note that land parcel information is frequently unavailable and thus

2 undertake a digitisation of historical data to create a temporal database of construction.

3 The difficulty with this kind of approach is the manual effort required to complete the

4 digitisation, in this case stated to be 30-40 hours for 1000 footprints.

Automatic analysis of the footprint shape and context have also been

6 demonstrated as an approach for inferring construction period in the UK context.

7 Alexander et al (2009) report a high accuracy for a subset of the age bands evaluated by

8 applying different techniques to topographic map data. However, their work does not

9 consider the use of neighbourhood metrics, nor DSM data, which has become more

10 widespread in recent years, as we propose in our work.

11 Tooke et al. (2014) describe the application of a predictive modelling to LiDAR

12 data in order to infer building ages in Vancouver, Canada, also with the aim of

13 supporting energy modelling applications. Their approach extracted spatial metrics,

14 which are used within a random forests regression model, to predict building age.

15 However, their work does consider improving the accuracy of the prediction through

16 spatial reasoning and analysis of the inference certainty, as we propose.

Biljecki and Sindram (2017) investigate the extent to which 3D city models can

18 be used to determine building age. They experiment using different combinations of

19 predictors, of building age extracted from a 3D city model such as building height,

20 number of storeys, use and enclosed volume. Our work focuses on using 2D national

21 mapping agency topographic data and DSM as sources, rather than a 3D city model.

22 In summary, we identify that relatively few approaches to determining either

23 building age or construction period have been proposed. These existing methods

24 involve either: (i) exhaustive manual digitisation - an approach which is not scalable to

25 large urban areas; or (ii) rely on datasets that are not always available and do not 
1 consider the multiple sources of building and neighbourhood data available in the UK

2 context; or (iii) do not consider the location or spatial context of a building as part of

3 their inference process to assigning an age or construction period attribute.

4 We propose here the use of a supervised machine learning method to enable

5 automatic inference of building age for energy modelling purposes, based on a variety

6 of different types of geospatial data. We then develop and evaluate methods to analyse

7 the model predictions and improve inference through a neighbourhood assessment of a

8 building's adjacencies and relations.

\section{$9 \quad 3$ Method}

\section{$10 \quad 3.1 \quad$ Study area}

11 Our study area is the city of Nottingham, UK. The residential building stock of

12 Nottingham exhibits a range of housing styles across age bands commonly found in the

13 UK. Figure 1 shows the Nottingham City boundary, the sample locations for

14 undertaking cross-validation model selection and testing and the detailed area of the

15 neighbourhood sample (both discussed in further detail below). 


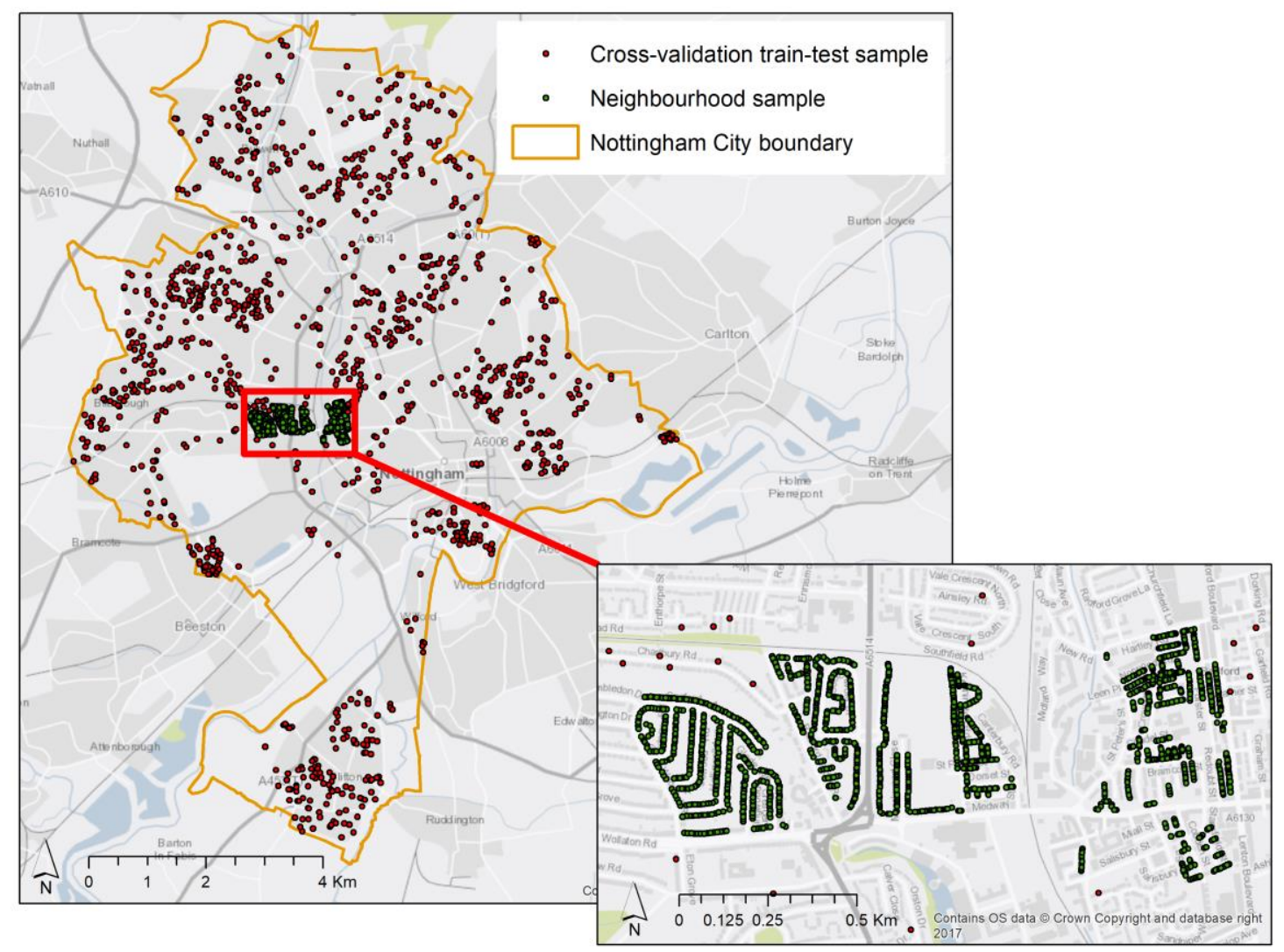

2 Figure 1. Nottingham city boundary area. Sample locations for cross-validation training and testing, and detailed

3 section showing 1457 houses used for neighbourhood area testing (see section 4 for further details).

\section{$4 \quad 3.2 \quad$ Data}

5 Topographic mapping, in the form of building footprints, Digital Surface Model (DSM)

6 and the statistical boundaries used by the UK national census were utilised for the

7 predictive model development. Topographic mapping forms the core data for analysis

8 from which building features were extracted.

We used Ordnance Survey (OSGB) MasterMap, which provides an accurate and

10 geometrically detailed representation of building footprints (Ordnance Survey 2017). To

11 create a dataset of only habitable building footprints (i.e no garages or sheds) we joined

12 the MasterMap data to AddressBase Plus (Ordnance Survey 2016), a UK database of

13 property address points, and removed polygons that were found to not have an address.

14 OSGB also provides a building heights database (Building Height Attribute) of

15 additional fields, which augment these footprints. These fields include a value for the 
1 building height as measured from ground-level, and ridgeline height of each footprint.

2 As MasterMap is a primarily 2.5D product when enriched with the building heights, a

$30.5 \mathrm{~m}$ spatial resolution DSM, created in-house by OSGB using photogrammetry of

4 aerial imagery, was used to provide additional 3D details. For census boundaries, the

5 Office of National Statistics portal provided 2011 Output Area polygons (ONS

6 Geography 2011).

\section{$7 \quad 3.2 .1 \quad$ Training and cross-validation data}

8 In order to train and test the supervised classification model, a city-wide sample of

9 buildings, their morphological and neighbourhood attributes and their age is required,

10 for ground-truth purposes. Five periods of construction including pre-1915, 1915 -

11 1944, 1945 - 1964, 1965 - 1979, and post-1980 were adopted for the classification.

12 These age bands reflect those found in the EHS, apart from a small difference in splits

13 between the first two periods (the EHS uses pre-1919 and 1919-1944), which was

14 necessary as these were the bands provided in the available source data. A spatial

15 dataset of the age and location of all local authority owned and built properties

16 (provided by Nottingham City Council) formed a starting point for creating this sample.

17 In Nottingham, the local authority has built over 50,000 homes since 1901, which represents over $40 \%$ of the city's housing stock. However, the dataset contained relatively few old properties (those built before 1915) and few modern properties (built

20 after 1980). To compensate for this sampling bias, we augmented the data with pre-

211915 and post-1980 properties drawn from a random household visit survey conducted

22 in 2014 and made available for this work (Long et al. 2015). To create a final training-

23 validation dataset, we randomly selected properties across each age band using

24 proportions obtained from a study that characterises the entire Nottingham housing

25 stock (Long et al. 2015). As this produced a sample of 1096 properties across the city - 
$180 \%$ was used for cross-validation model selection and training and $20 \%$ was used as a

2 validation dataset, as summarised in Table 1. The locations of this sample are shown in

3 Figure 1.

4

Table 1. Sample descriptions, class sizes, and splits used for training, validation and testing.

\begin{tabular}{|c|c|c|c|c|c|c|c|}
\hline $\begin{array}{c}\text { Sample } \\
\text { description }\end{array}$ & $\begin{array}{l}\text { pre- } \\
1915\end{array}$ & $1915-$ & $1945-$ & $1965-$ & $\begin{array}{l}\text { post- } \\
1980\end{array}$ & Split & Usage description \\
\hline \multirow[b]{2}{*}{$\begin{array}{l}1096 \text { points - } \\
\text { city-wide sample }\end{array}$} & \multirow[b]{2}{*}{229} & \multirow[b]{2}{*}{280} & \multirow[b]{2}{*}{259} & \multirow[b]{2}{*}{220} & \multirow[b]{2}{*}{108} & $80 \%$ & $\begin{array}{c}\text { Cross-validation model selection, hyper-parameter } \\
\text { tuning, learning curve training data, final model training. }\end{array}$ \\
\hline & & & & & & $20 \%$ & $\begin{array}{c}\text { Test set for user and producer accuracy calculation, } \\
\text { selected CV model learning curve testing and trained CV } \\
\text { model testing. }\end{array}$ \\
\hline $\begin{array}{l}1457 \text { points - } \\
\text { neighbourhood } \\
\text { sample }\end{array}$ & 301 & 463 & 363 & 203 & 127 & $100 \%$ & $\begin{array}{l}\text { Neighbourhood test set for post-classification spatial } \\
\text { reasoning testing }\end{array}$ \\
\hline
\end{tabular}

5

\section{$6 \quad$ 3.2.2 Neighbourhood testing sample}

7 In addition to the city-wide training and cross-validation samples, evaluation of the

8 predictive model and subsequent post-classification spatial analysis utilised a specific

9 residential area sample. The area was chosen due to its inclusion of residential

10 properties across all age bands. Non-residential buildings and blocks of flats were

11 removed from this sample and no buildings within the area were included in the city-

12 wide training and cross-validation samples (to ensure effective validation). This created

13 a sample of 1457 properties for the neighbourhood evaluation, the locations of which

14 are shown in Figure 1.

\section{$15 \quad 3.3 \quad$ Predictors}

16 To infer age, features based on building (see section 3.3.1) and neighbourhood

17 characteristics (see section 3.3.4) were derived from the vector-based topographic 
1 mapping, DSM and census boundary datasets. Our experiments utilised fifteen

2 predictors in total, summarised in Table 2.

3

Table 2. Features used for characterizing the construction period of buildings

\begin{tabular}{|c|c|}
\hline Predictor & Description \\
\hline Area $(m)$ & Area of building footprint \\
\hline Perimeter $(m)$ & Length of a building outline \\
\hline NPI & Normalized Perimeter Index \\
\hline footprintVertices & A count of the number of vertices that make up the building footprint \\
\hline relHEaves $(m)$ & Height of building eaves relative to the ground \\
\hline $\operatorname{relHMax}(m)$ & Maximum height of building relative to the ground \\
\hline roofPitchMean $(m)$ & Average slope of the roof pitch DSM pixels falling within the building footprint \\
\hline roofHeightStdDev $(m)$ & Standard deviation of DSM pixels falling within the building footprint \\
\hline builtForm & The building is detached, semi-detached, terraced, end-terraced or complex. \\
\hline numberInBlock & A count of the number of buildings that are in a block (i.e. are topologically related) \\
\hline sharedPerimeter $(m)$ & Length of the building outline shared between touching building footprints \\
\hline non-sharedPerimeter $(\mathrm{m})$ & Length of the building outline not shared between touching building footprints \\
\hline exteriorRatio & Ratio of the length of the exposed building outline divided by the total footprint perimeter \\
\hline builtUpAreaRatio & A ratio of built-up area size $(m)$ to the size $(m)$ of the census Output Area \\
\hline voronArea $(m)$ & Area of the Voronoi parcel estimated around the building's centroid \\
\hline
\end{tabular}

\section{$5 \quad$ 3.3.1 Building footprint geometry}

6 The area of the building footprint $\left(\mathrm{m}^{2}\right)$ is computed as this can provide some indication

7 of its age. For example, over the last 30 years in the UK and the US contexts, old (e.g.

8 pre-1919) houses are noted to be small relative to modern standards, and a tendency of

9 increasing house size over time has been documented (Brown and Steadman 1991, 
1 Wilson and Boehland 2005). However, in more recent years, this is also paralleled by

2 construction of homes with smaller floor areas (RIBA 2015).

3

4 6 perimeter.

We utilise a measure of polygon complexity to characterise the shape of the building footprints. The Normalised Perimeter Index (NPI) has been shown to help with inferring building heights (Biljecki et al. 2017) and identification of building types (Wurm et al. 2016), which in turn can provide evidence for a particular construction period. NPI provides a characterisation of the compactness of the building footprint shape and is calculated using $\frac{2 \sqrt{a \pi}}{p}$ (where $a$ and $p$ are the footprint area and perimeter respectively). Example measures of NPI are shown in Figure 2. As an additional measure of shape complexity, the number of vertices that make up the footprint shape was also recorded.
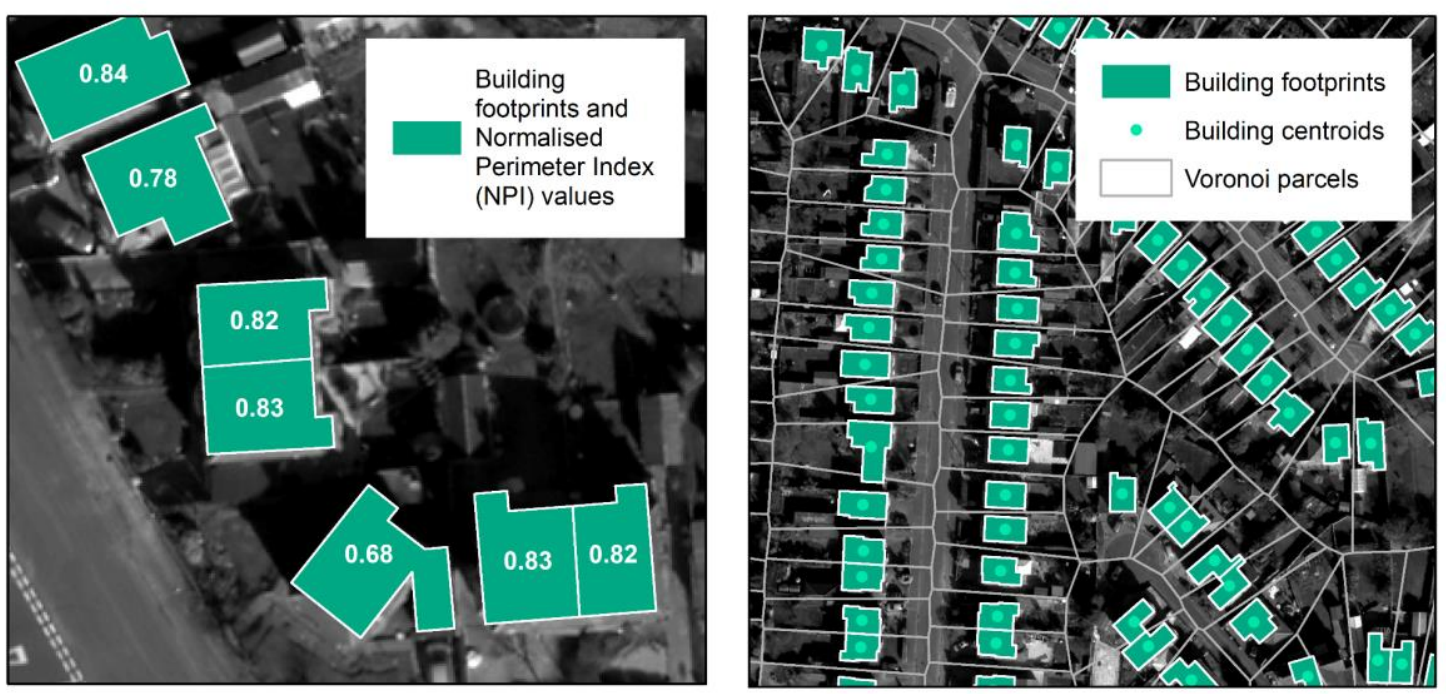

17 Figure 2. Example building footprints with their Normalised Perimeter Index (NPI) values (left) and example

18 footprint centroids (right). The Voronoi parcels depicted are discussed in further detail in section 3.3.4. 


\section{$1 \quad 3.3 .2$ Building roof and height details}

2 The three-dimensional form of buildings is an expression of their architectural style and

3 can thus provide a meaningful indication of their age. We use two measures relating to

4 the building height $(\mathrm{m})$, namely relative eaves height (approximately ground level to

5 eaves height) and maximum height (approximately ground level to the highest feature

6 including dormers or chimneys). This is sourced from the OSGB MasterMap building

7 height attributes. In addition to these height measures, we use two measures relating

8 directly to the roof geometry. The first is to estimate the pitch of the roof by calculating

9 the mean slope (degrees) within each building footprint. The second aims to quantify

10 complexity in the roof by calculating the standard deviation of the height values (m)

11 within each building footprint. These two roof predictors are sourced from the DSM.

12 Examples of these predictors are shown in Figure 3.
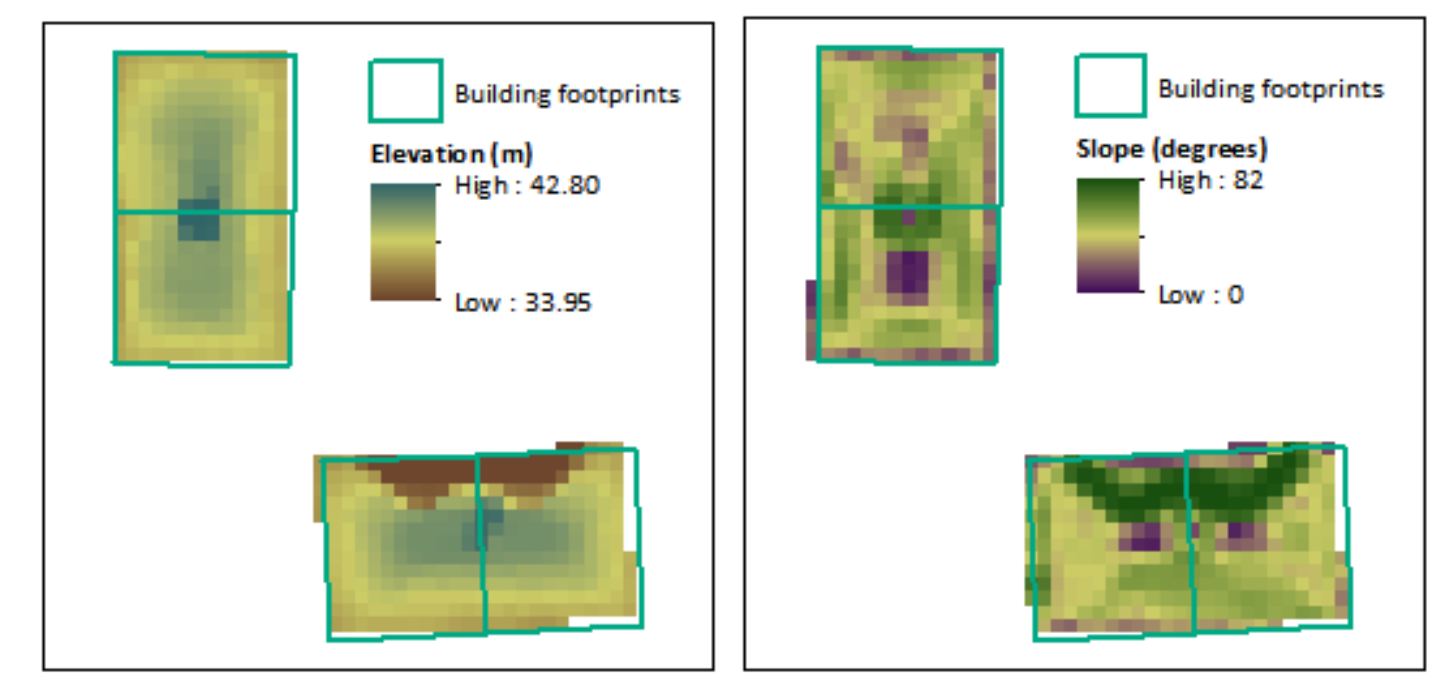

13

14 Figure 3. Roof elevation used to derive standard deviation of roof heights in order to infer roof complexity (left) and 15 roof slope, used to derive mean roof pitch (right).

18 We include several features relating to the topology of the property. The first is the built 
1 form (i.e. whether the construction is terraced, end-terraced, semi-detached or

2 detached). This feature was determined using a topological query of each footprint

3 where the number of neighbours determines the form, (see Figure 4, left). We follow the

4 implementation of Beck et al (2018) to first classify buildings based on touching

5 neighbours (i.e. 0 is detached, 1 is semi-detached or end-terraced, 2 is mid-terraced),

6 and then separate the end-terraced buildings into their own class where the number

7 properties in a whole block of touching polygons is greater than two. In addition to the

8 built form, a block level metric defining the number of buildings in a topological block

9 (i.e. the number of buildings that form a group of buildings that are touching) is used

10 (see Figure 4, right).
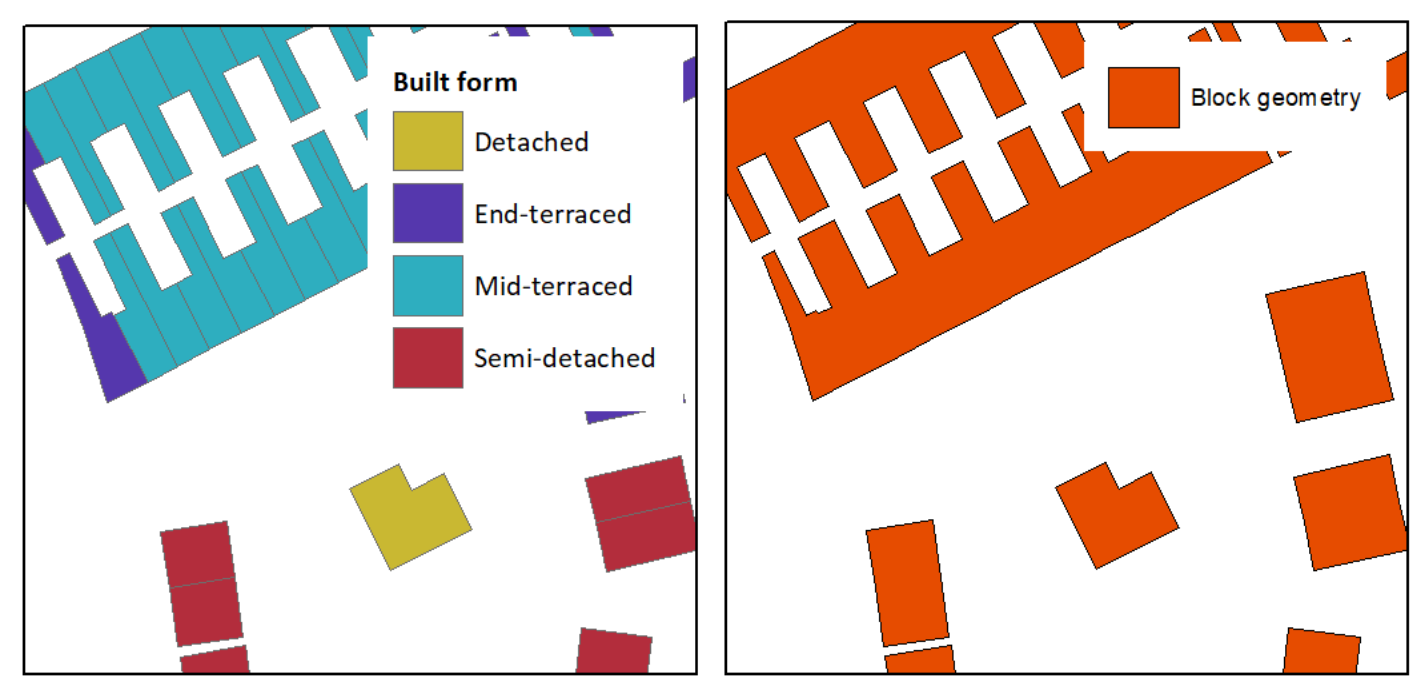

13 Figure 4. Built form classification showing detached, end-terraced, mid-terraced and semi-detached properties (left)

14 and block level geometry used to determine number of buildings that are touching (right).

15 Two measures pertaining to the lengths $(\mathrm{m})$ of the building's outline that are touching

were calculated. These include the length of the shared wall and the length of the

17 exposed wall. Lastly, the exposed wall length divided by the perimeter was computed in

18 order to define an exterior wall ratio value. 


\subsubsection{Neighbourhood attributes}

The density of buildings can give a particular character to a neighbourhood or

4 local area. To provide an indication of the degree to which the neighbourhood is

5 urbanised, a measure of built-up area was calculated. Here we adopt the census Output

6 Area (OA) as a neighbourhood zone to which a built-up area statistic can be attached.

7 The built-up area is defined as the total area of buildings (both residential and non-

8 residential) within each OA, normalised by area of the OA. The amount of open space

9 surrounding a building can also provide an indication of the type of development within

10 an area. For example, neighbourhood details based on the Voronoi relations have been

11 shown to help identify usage and occupancy of buildings (Huang et al. 2013). The lot

12 area (i.e. property parcel) was found to be a strong predictor of age in previous work

13 (Tooke et al. 2014). We use the Voronoi diagram constructed over the centroids of each

14 footprint that has an address (thus including both residential and non-residential

15 properties) to estimate a land parcel surrounding a building, and calculated its area $\left(\mathrm{m}^{2}\right)$.

16 An illustration of the Voronoi parcels is shown in Figure 2.

\section{$17 \quad 3.4 \quad$ Model implementation}

\section{$18 \quad 3.4 .1 \quad$ Predictive model}

19 We implement the statistical model for learning the predictors as a random forest multi-

20 class classification problem. Random forests are a widely used ensemble-based

21 supervised learning method suitable for both classification and regression problems

22 (Breiman 2001). Breiman (2001) demonstrates that random forests are robust to noise,

23 are computationally efficient to train, handle both categorical and continuous data, 
1 provide a measure of variable importance and unlike Support Vector Machines and

2 multi-layer perceptron types of learning models, have the advantage of estimating class

3 probability when used for inference. In summary, as an ensemble method random

4 forests extend a decision tree-type estimator. Decision trees model the classification

5 task as a tree defined as recursive binary splits of the predictor variables at important

6 values. To determine the splitting, a measure of the node (leaf) impurity is required,

7 typically using either entropy or Gini importance. We use the Gini importance to

8 determine splitting which calculates how much a feature reduces the impurity resulting

9 from the split. Decision trees offer an appealing method for learning due to their

10 invariance to transformations on the predictors (thus obviating the need for feature

11 normalisation). They perform an internal feature selection as part of their training

12 procedure, yet they also suffer from high variance and perform poorly in comparison to

13 other estimators (Hastie et al. 2009). Tree-based bootstrap aggregation or bagging

14 extends the decision tree approach by training a set of trees on subsets of the data

15 derived from bootstrap sampling (sampling with replacement). During inference, the

16 average prediction is taken across all trees, yielding a more powerful estimator than a

17 single model. In random forests, the splitting of each tree is based on a random sample

$18(\mathrm{~m})$ of the available predictors, thus de-correlating individual trees and making the

19 bagged average prediction more reliable and less variable (James et al. 2007).

20 We adopt the random forest package of the Apache Spark (v2.1) distributed computing

21 framework (spark.apache.org), which adopts the Breiman (2001) implementation and

22 enables rapid, parallelised model testing and evaluation (Zaharia et al. 2010). 


\section{1}

2 Most statistical learning methods, including random forest classification, operate on a

3 model where labelled vectors of feature values are used for training and testing. When

4 applying a trained model for practical purposes, the model inference does not take

5 account of the spatial nature of the phenomena in question - in this case an estimated

6 class of building age. For the case of building age inference, we identify that an

7 automatic analysis of the classifier prediction, which adopts some form of spatial

8 reasoning about the scene, can improve upon per-building estimates made by the

9 classifier alone. For example, in the case of two semi-detached houses linked by an

10 adjoining wall, it is highly probable that such houses were built at the same time. The

11 same is also probable in the case of a block of terraced houses. Furthermore, this spatial

12 autocorrelation of the building age is not limited to structures that are touching. Housing

13 construction is likely to have been undertaken by developers who build a set of houses

14 across a block or plot of land, and this means clusters of nearby similarly aged

15 residential buildings can often be identified in suburban and city centre residential areas.

16 We test three approaches to exploiting spatial autocorrelation to improve our

17 building age prediction. These techniques utilise the probability value of each age class

18 produced by the random forest classifier. Typically, when conducting inference with a

19 random forest classifier, the class with the highest probability is assigned for a single

20 query data point. Instead, we aggregate the per-building class prediction probabilities

21 across groups of buildings. Determining these groups depends upon defining spatial

22 relations between the objects. Here we use both touching and nearness.

Our first approach is to take the mean of each class prediction across all

24 buildings in a block. Then, the class with the maximum mean probability is assigned to

25 each building in the block. 
The second approach extends the first to buildings that are near to each other.

2 Using the same Voronoi diagram, described above in section 3.3.4, we define near

3 neighbour relations between buildings. These nearby buildings correspond to the nodes

4 of the Delaunay triangulation dual graph of the Voronoi diagram. Using the relations

5 defined by this graph, for each building we can take the mean of the class probabilities

6 over both itself and its neighbours, and then assign the class with the maximum mean

7 probability to that building.

The third approach extends the second approach to an energy minimization

9 problem, which is solved using graph cuts (Boykov et al. 2001, Boykov and

10 Kolmogorov 2004, Kolmogorov and Zabih 2004, Fulkerson et al. 2009). The graph cuts

11 method has been widely used in image segmentation and smoothing applications, where

12 spatial coherence over pixels helps to identify the features depicted. Schindler (2012)

13 reviews its application in land cover remote sensing classification, for example. The

14 method generalises to other graph structures (non-imagery) problems such as grouping

15 of buildings for 3D city model simplification (Wang et al. 2015). The graph cuts

16 method is an approach to finding the minimum cut or maximum flow path across a

17 graph. It can be used to find the set of labels that minimizes an energy function

18 comprised of a term corresponding to the observed data (known as unary) and a term

19 corresponding to an adjacency graph (known as pairwise or boundary).

$$
E(L)=U(L)+\lambda B(L)
$$

21 Where $L$ is the labelling for the nodes, $U(L)$ is the unary term, $B(L)$ is the pairwise term,

22 and $\lambda$ is a weighting factor (which here is set to 15). We adopt similar energy functions

23 to the image segmentation problems described by Fulkerson et al. (2009) and

24 Hernandez-Vela (2012), using the confidence in the classifier predictions. In particular, 
1 we use the random forest prediction probabilities of each class to define the unary term:

$$
\mathrm{U}(L)=\sum_{i \in \mathcal{P}}-\log \left(c_{i}\right)
$$

3 Where $\mathcal{P}$ is the set of nodes and $c_{i}$ is the vector of age class probabilities for node $i$

$4 \quad$ which has been inferred by the classifier. In image segmentation problems, the pairwise term is usually defined as a 4-

6 pixel or 8-pixel neighbourhood around each pixel. In our case, however, we use the 7 adjacencies defined by the Voronoi neighbours to define the graph. Iterating over the 8 adjacencies allows us to define a cost for each edge based on the Euclidean n-space 9 distance between the class probabilities of the corresponding points.

$$
B(L)=\sum_{\{i, j\} \in \mathcal{N}}\left\|x_{i}-x_{j}\right\| \cdot \delta\left(L_{i} L_{j}\right)
$$

$$
\delta\left(L_{i} L_{j}\right)=\left\{\begin{array}{c}
1 \text { if } L_{i} \neq L_{j} \\
0
\end{array}\right.
$$

12 where $x$ is the vector of class probabilities for a node $(i, j)$. Here the smoothing term

$13 \delta\left(L_{i} L_{j}\right)$ fulfils the role of an indicator function which encourages similarity in the 14 building labelling.

\section{Results}

\subsection{Model selection and tuning}

17 We test various combinations of predictor to investigate the model accuracy obtainable

18 in different scenarios of data availability. Furthermore, random forests require

19 specification of several hyper-parameters for effective training and inference. The

20 number of predictors selected for determining tree splits $(m)$, the number of trees that

21 make up the forest, the maximum depth to which each tree may branch and the binning 
1 used when discretizing continuous features are important in determining the

2 performance of the classifier. Although large numbers of trees can improve

3 performance, studies have shown that such improvements can level off (Hastie et al.

4 2009, Oshiro and Perez 2012) while the time to fit the model increases. Simultaneously,

5 the depth of the tree can improve predictive performance but can increase overfitting.

6 To evaluate each of our models and the corresponding hyper-parameters, we applied a

7 grid search and cross-validation evaluation. To determine $m$, from all available

8 predictors $(p)$, we use $m=\sqrt{p}$, as suggested by Breiman (1984) in all model cases. For

9 the remaining hyper-parameters, we used a 5-fold cross-validation grid search on $80 \%$

10 of features randomly selected from the city-wide sample, in order to identify values

11 close to optimal, according to a particular evaluation metric. Given our multi-class

12 prediction problem, we use accuracy for the metric, which reports the number of true

13 positives normalised by the number of data points. For the grid search parameters,

14 maximum tree depths of 3, 4 and 5, maximum binning values of 35, 45, 60 and 80 and

15 forests of size $30,40,60,80,100$ and 120 were used.

16 Table 3 illustrates combinations of features used and the accuracy for the best

17 performing model, as identified by the grid search. Table 3 indicates that relatively

18 strong classifier performance is achievable even with a limited set of predictors. For

19 example, Model 6, which excludes the neighbourhood features, achieves $74 \%$ cross-

20 validation accuracy and Model 8 , which excludes the DSM derived metrics, achieves

$2172 \%$.

22 The model using all features achieved the highest cross-validation accuracy (75\%), with

23 hyper-parameters of 40 trees, a maximum depth of 5 and a maximum binning of 60 .

24 This model was selected for further analysis. For practical evaluation of the classifier we use confusion matrices, see section 4.2 and 4.3 . 
Table 3. Models with different combinations of predictors (features) and cross-validation accuracy.

\begin{tabular}{|c|c|c|c|c|c|c|c|c|c|}
\hline \multirow[t]{2}{*}{ Predictor } & \multicolumn{9}{|c|}{ Model\# } \\
\hline & 1 & 2 & 3 & 4 & 5 & 6 & 7 & 8 & 9 \\
\hline $\operatorname{Area}(m)$ & o & o & o & o & o & o & o & o & o \\
\hline Perimeter $(m)$ & & o & o & o & o & o & o & o & o \\
\hline$N P I$ & & & o & o & o & o & o & o & o \\
\hline footprintVertices & & & o & o & o & o & o & o & o \\
\hline relHEaves $(m)$ & & & & o & o & o & & o & o \\
\hline relHMax $(m)$ & & & & o & o & o & & o & o \\
\hline roofPitchMean $(m)$ & & & & & o & o & & & o \\
\hline roofHeightStdDev $(m)$ & & & & & o & o & & & o \\
\hline builtForm & & & & & & o & o & o & o \\
\hline numberInBlock & & & & & & o & o & o & o \\
\hline sharedPerimeter $(m)$ & & & & & & o & o & o & o \\
\hline non-sharedPerimeter $(m)$ & & & & & & o & o & o & o \\
\hline exteriorRatio & & & & & & o & o & o & o \\
\hline builtUpAreaRatio & & & & & & & o & o & o \\
\hline voronArea $(m)$ & & & & & & & o & o & o \\
\hline Overall CV Accuracy & 0.46 & 0.6 & 0.66 & 0.69 & 0.72 & 0.74 & 0.7 & 0.72 & 0.75 \\
\hline
\end{tabular}

2 Plotting the learning curve of the classifier allows for identification of bias and

3 variance, and thus the trade-off between underfitting and overfitting, in the model.

4 Furthermore, the learning curve shows the effect of sample size on the efficacy of

5 prediction. Using the best performing model (Model 9) and hyper-parameters identified

6 from the 5-fold cross validation above, iterative increases of 100 data points for the

7 training set size was tested. For this evaluation, $80 \%$ of the city-wide sample was used

8 for training and the remaining $20 \%$ was used for testing, as shown in Table 1 . The

9 training and testing accuracy (i.e. $\operatorname{accuracy}(y, \hat{y})=\frac{1}{n} \sum_{i=0}^{n-1} 1\left(\widehat{y_{l}}=y_{i}\right)$ where $\widehat{y}_{l}$ is the 
1 predicted value and $y_{i}$ is the corresponding true value) were computed for each sample

2 to create the learning curve for the model shown in Figure 5. We can see that the testing

3 accuracy appears to plateau at approximately $77 \%$, close to the model's training

4 accuracy of approximately $80 \%$.

\section{$7 \quad 4.2 \quad$ Model performance, feature importance and cross-validation}

8 The feature importance metric, shown in Figure 6, is exposed by the Apache Spark

9 classification module and is computed according to the average of its importance (in our

10 case, Gini importance) across all trees in the ensemble, with the resulting vector

11 normalized to sum to 1 (Hastie et al. 2009). Figure 6 shows the measures of footprint

12 complexity (NPI), footprint size (footprintArea) and average roof pitch (roofPitchMean)

13 are the strongest predictors for construction period. The measure of built-up area

14 (builtUpAreaRatio) and perimeter also appear to be useful discriminators. In contrast,

15 the number of vertices (footprintVertices), the measure of the roof shape complexity

16 (roofHeightStdDev), the roof eaves height (relHEaves) and the estimated size of the

17 land around the property (voronArea) are relatively poor as indicators of age.

18 A confusion matrix of the model performance offers insight into the predictive power

19 across different age classes. Figure 7 shows the confusion matrix (row-wise normalised)

20 calculated on the $20 \%$ city-wide test set. The results show effective identification of the 
1 pre-1915(82\%), $1945-1964(88 \%)$ and $1965-1979(78 \%)$ age bands. The classifier

2 scores worse on $1915-1944(67 \%)$ and post-1980 (50\%) and there is a notable

3 discrepancy with the differentiation between post-1980 and pre-1915 properties; this

4 latter reflecting the relative scarcity of data in these categories.

5 In addition to the overall accuracy of $77 \%$, we compute user's accuracy (precision) as

$670 \%$, and producer's accuracy (recall) of 73\%. The kappa statistic, which takes into

7 account chance matches between the predicted and true classes, of 0.7 indicates

8 substantial agreement (Landis and Koch 1977).
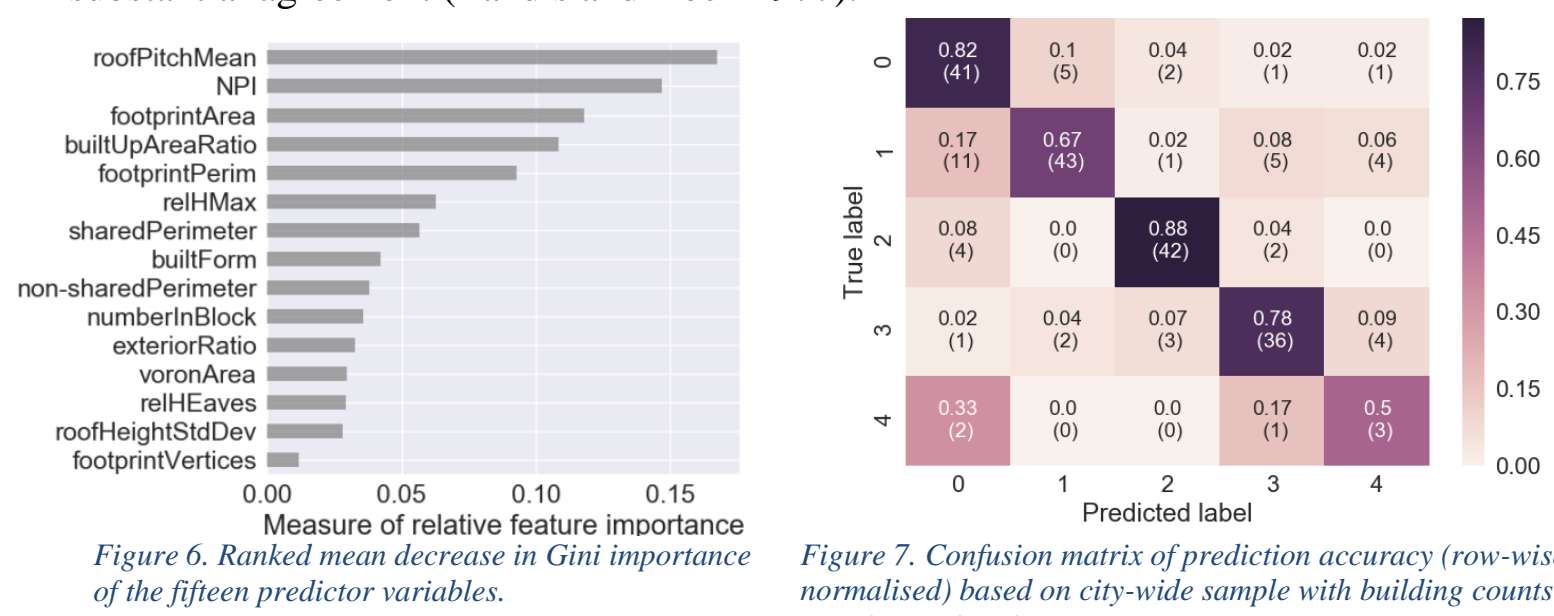

Figure 7. Confusion matrix of prediction accuracy (row-wise normalised) based on city-wide sample with building counts per class in brackets

\section{$9 \quad 4.3 \quad$ Neighbourhood specific sample}

10 Here we evaluate the classifier on the neighbourhood sample of 1457 houses, and apply

11 the techniques described in section 3.4.2 to improve upon the age band estimate

12 provided by the predictive model alone.

13 Table 4. User's and producer's accuracy (UA and PA), overall accuracy (OA) and kappa statistic.

\begin{tabular}{c|c|c|c|c}
\hline Model & $\boldsymbol{U A}$ & $\boldsymbol{P A}$ & $\boldsymbol{O A}$ & Kappa \\
\hline Prediction only & 57 & 57 & 64 & 0.54 \\
\hline Block averaging & 59 & 58 & 65 & 0.55 \\
\hline Near-neighbour averaging & 61 & 60 & 68 & 0.59 \\
\hline Graph-cuts label optimisation & 56 & 61 & 72 & 0.64 \\
\hline
\end{tabular}


1 Table 4 presents the different accuracy metric of the neighbourhood sample, and Figure

28 shows the confusion matrices. These show the prediction model estimate (overall

3 accuracy $=64 \%$ ), block averaged predictions (overall accuracy $=65 \%$ ), near-neighbour

4 (Voronoi) averaging (overall accuracy $=68 \%$ ) and graph-cuts label optimisation

$5 \quad$ (overall accuracy $=72 \%)$. Considering the user's and producer's accuracy and kappa,

6 we see that the different methods of adjusting the predictions generally improve the

7 performance. However, there is a notable drop in user's accuracy (precision) when

8 applying the graph-cuts approach; though this is offset by an increase in producer's and

9 overall accuracy. Considering the confusion matrices in Figure 8, this is due to an

10 increase in the misclassification of post-1980 properties as pre-1915.
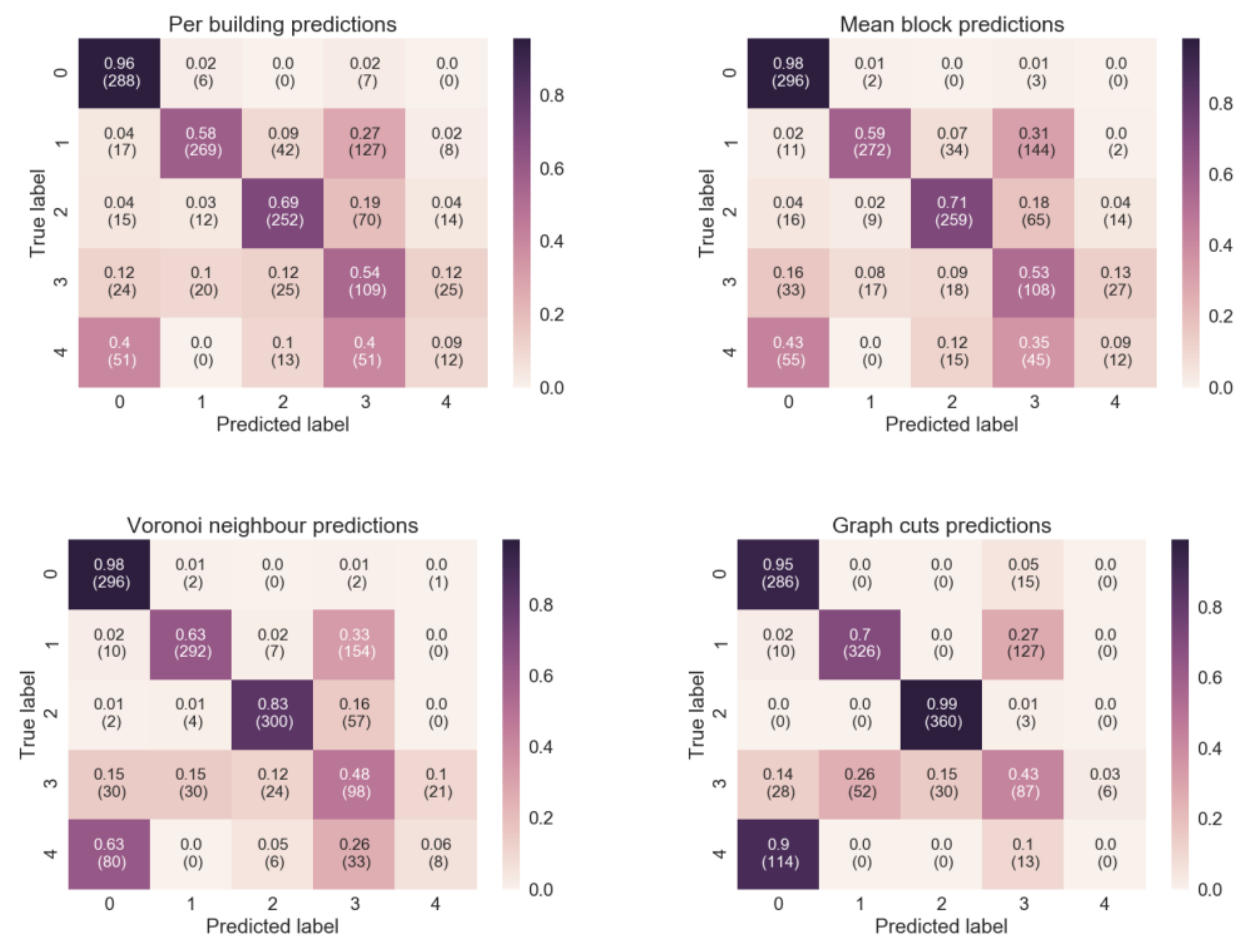

14 Figure 8. Normalised (row-wise) confusion matrices for the neighbourhood sample. Prediction model estimate (top15 left), averaged prediction confidences by building block (top-right), averaged prediction confidences by Voronoi 16 adjacencies (bottom-left), graph-cut label optimisation using Voronoi adjacencies (bottom-right).

In Figure 9 and Figure 10 we show maps of the ground-truth and predicted 
1 per-building inference, we can see that the method appears to capture a reasonable

2 overall distribution of property ages across the scene. However, some areas such as the

3 housing found at the centre of the map are estimated incorrectly. The map of block

4 averaged predictions is seen to improve the accuracy in some areas, particularly where

5 long pre-1915 housing terraces include a property with an anomalous age. The near-

6 neighbour averaging and graph-cuts label optimisation smooth the predicted ages,

7 particularly in the Western and Eastern sides of the map. The graph-cuts approach in

8 particular, which scores highest overall, does however incorrectly group many buildings

9 together into the pre-1915 band, while conversely correctly expanding the cluster of

10 1946-1964 detached properties.

11 

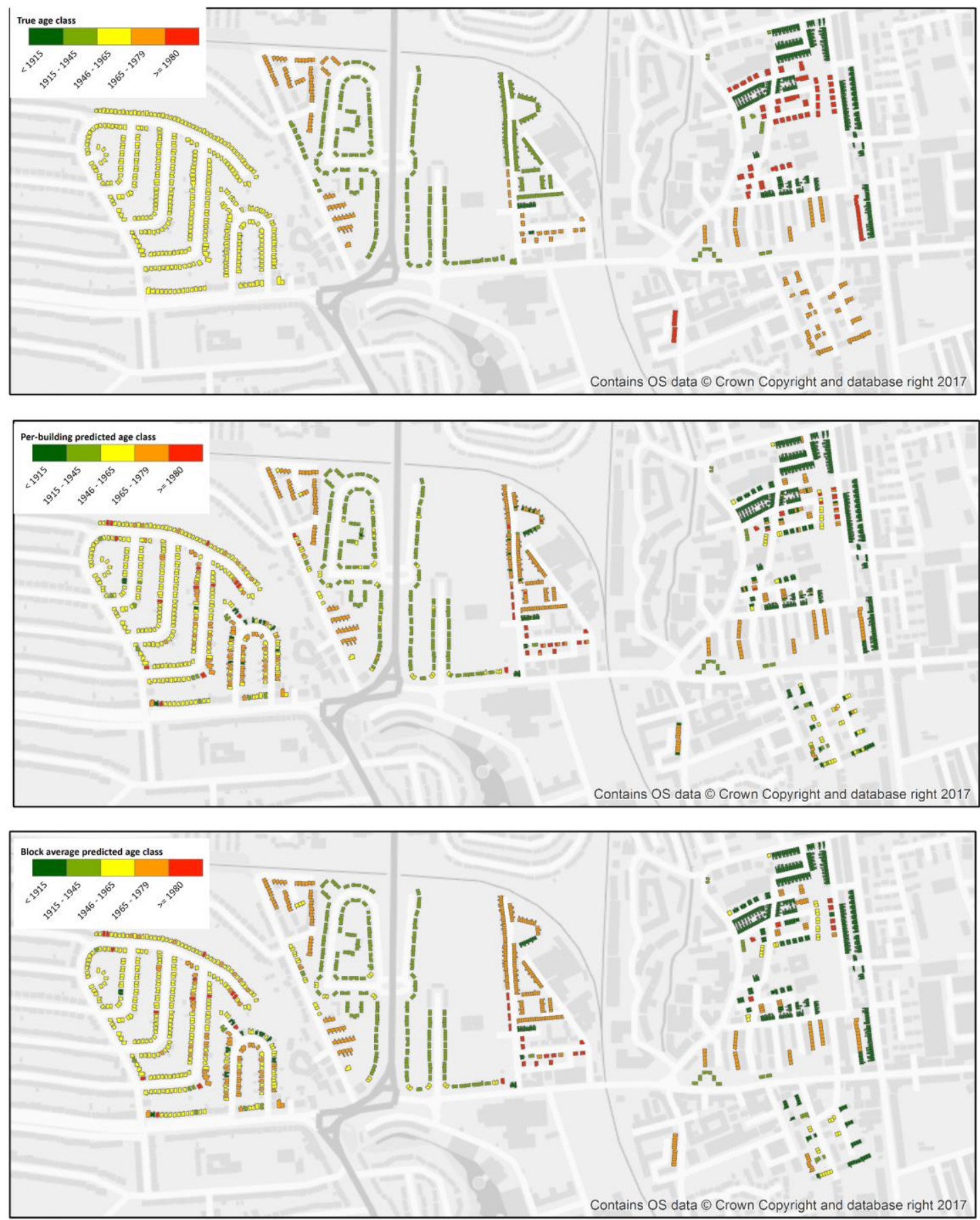

2 Figure 9. Neighbourhood sample showing true age class (top), per-building predicted age class (middle) and block averaged predicted age class (bottom). 


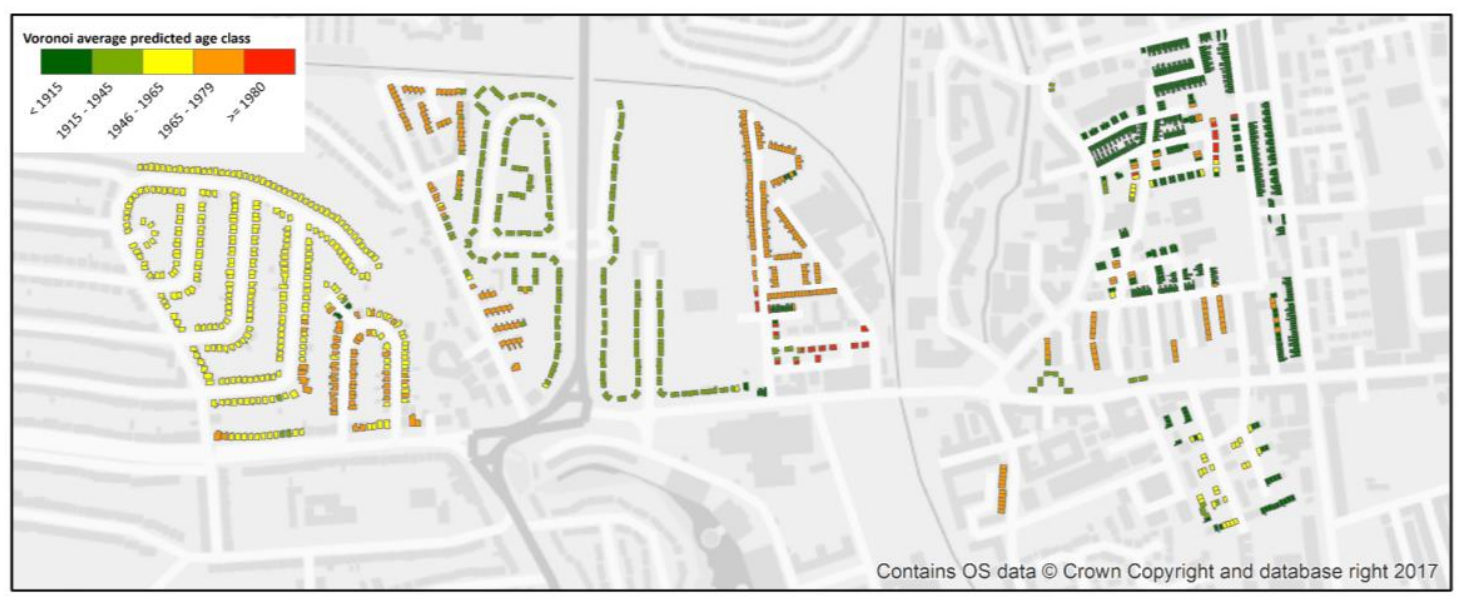

2

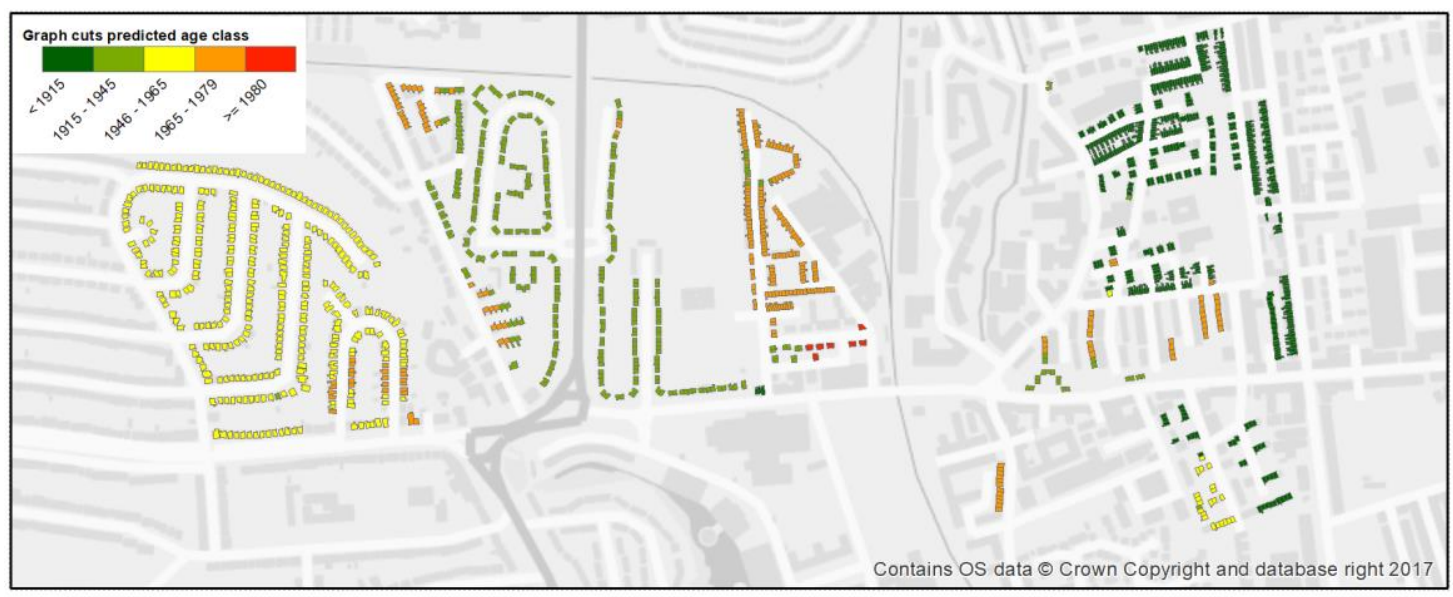

Figure 10. Predicted age class based on Voronoi average (top) and graph-cuts label optimisation (bottom)

\section{Discussion}

In this work, we demonstrated the assignment of a construction period of a residential property given certain evidence that can be automatically extracted from maps. Using geometric measures of the building footprint, a digital surface model and neighbourhood statistics as a source of variables, a predictive model is shown to achieve reasonable accuracy ( 5 -fold cross-validation $=75 \%$, and $77 \%$ test accuracy) and this estimation can effectively be improved through an automatic post-classification analysis. A data-driven model for estimating building age will never achieve perfect accuracy. As-built survey with extensive manual work is the only absolutely reliable way to assign all buildings a correct construction period. However, as this is prohibitively expensive, a data-driven approach can serve as a useful tool for energy 
1 modelling practitioners undertaking city-wide analysis, and provide guidance on the

2 locations of properties where the construction period has been inferred with a high

3 degree of confidence.

Analysing the incorrect predictions made by the classifier (see Figure 6)

5 indicates that a large proportion are within one age band of the true construction period.

6 This degree of error is not likely to have a significant impact, estimated to be less than

$7 \quad 10 \%$ based on analysis of gas usage by dwelling age reported in the Homes Energy

8 Efficiency Database statistics (Hamilton et al. 2013). However, in the relatively

9 unabundant case of modern buildings (post 1980), over 30\% are incorrectly assigned as

10 being constructed before 1915 . This would lead to substantial errors when modelling the

11 energy use of these buildings, which are generally less efficient than modern properties

12 (Hamilton et al. 2013). In general, the performance of the classifier on predicting ages

13 of modern building is shown to be challenging. The wide variation in age prediction for

14 these buildings is likely due to their sparsity in the training set, leading to insufficient

15 discrimination in the model for effective inference. Further work could incorporate a

16 larger sample of modern buildings. In the UK, at least, poor performance for this age

17 band may be partly ameliorated by the recently released Valuation Office Agency price

18 paid data (landregistry.data.gov.uk), which enables identification of properties

19 constructed during or after 1995. This dataset could form a useful set of additional

20 training data or be used to automatically correct inferred classes.

21 Further investigation of the contribution of individual features may also help.

22 Research has suggested that estimates of feature importance in random forests may be

23 biased when variables are of mixed type or scale (Strobl et al. 2007). Therefore,

24 analysis of predictor relevance could be undertaken by adopting permutation 
1 importance, rather than the Gini criterion, or by adopting an alternative implementation

2 of the random forest (e.g. conditional random forests).

One aspect that we note in this work is the difference in performance for the

4 trained predictive model validated against city-wide data versus the neighbourhood

5 validation. The likely cause for this variation lies in the relatively unusual style of 1920s

6 properties in the centre of the neighbourhood area which are likely to be under-

7 represented in the training data, resulting in their misclassification as $1965-1979$

8 properties. Recall that there were no training sample points within the neighbourhood

9 area boundary in order to avoid biasing the predictive model and to effectively evaluate

10 post-classification improvement. In practical deployment of this approach, a random

11 sample across all city areas would be recommended.

With respect to the label optimisation based on graph cuts, we highlight that

13 alternative or more detailed cost functions might further improve the approach. For

14 example, the pairwise cost might utilise a more complex graph structure of the spatial

15 relations, such as $1^{\text {st }}$ order and $2^{\text {nd }}$ order adjacencies, and a spatial Euclidean distance

16 could be incorporated to weight neighbours differently based on their proximity. Cross-

17 validation, expert judgement or a prior measure of spatial autocorrelation (such as

18 Moran's I) could inform such a distance weighting. Similarly, the label cost $\delta\left(L_{i} L_{j}\right)$

19 could encode further knowledge about the feasibility of grouping certain buildings, such

20 as relaxing the constraints for particular ages to appear adjacent to each other (e.g. if

21 modern re-developments are particularly common in old sectors of a city). Lastly, it

22 should be noted that the overall weighting of the pairwise term $\lambda$ may be adjusted for

23 particular scenes based on expert prior knowledge. For example, a higher weight can be

24 used to enforce larger groupings and vice versa. 
2 forest classifier prediction probabilities. However, it is important to note that similar

3 label optimisation approaches could be applied to another type of machine learning

4 classifier, providing that an assessment of class certainty or probability associated with

5 the inference is part of the predictive model. For example, many other ensemble

6 methods (e.g. AdaBoost) calculate probabilities as part of their inference (Breiman

7 2001); artificial neural network models can be trained to generate class probabilities

8 (Bishop 1995) and Bayesian networks provide a degree of belief associated with a class

9 prediction (Jensen 1996).

10 The work presented here utilised a sample of local properties for training the

11 classifier. For application in other urban areas, a local sample would likely yield the

12 best performance. However, a further interesting avenue for research could compare the

13 performance of a local sample versus a model trained using a national, regional or

14 alternative sample area. This may lead to greater insights on generality of features to

15 other areas.

16 We tested models using features extracted from geospatial data sources that are

17 readily available in the UK. However, our approach could in principle be applied in

18 other countries where equivalent data is available from mapping agencies or perhaps

19 from Volunteered Geographic Information or crowdsourcing campaigns. In urban areas,

20 OpenStreetMap (OSM) building footprints can have a high-level of completeness and

21 geometric similarity to authoritative map data, although commonly lack attribute level

22 data (Fan et al. 2014). Use of this data to infer building age offers a promising line of

23 research and a potential method of OSM enrichment. Similarly, further work on using

24 alternative features, which might be found in other countries could be undertaken. For

25 example, city models can include volume or gross-floor area attributes based on true 3D 
1 building geometry. In this work, singular height values per property are used but these

2 may overestimate sizes of properties where the building plan varies across different

3 floor levels.

\section{Conclusion}

In this paper, we developed and tested an automatic two-stage methodology for

6 inferring the age of residential buildings for the purposes of energy modelling, using

7 geospatial data that is readily available in the UK context. We use measures of building

8 shape and neighbourhood characteristics that form predictors in a random forest

9 classifier for estimating a per-building age class band. Both raster (a high-resolution

10 Digital Surface Model) and vector-based map data (topographic mapping) are used to

11 derive these measures. The predictive model achieves a cross-validation accuracy score

12 of $75 \%$ and validation set score of $77 \%$. We also describe and test methods of

13 processing per-building age inferences using the topology and spatial relations of the

14 urban structure to enforce likely assumptions on the classifier's prediction. We show

15 that correct assignment of construction periods can be improved by up to 8 percentage

16 points through post-prediction optimisation of the class labels.

\section{$17 \quad 7 \quad$ Acknowledgements}

18 The OS MasterMap data were made available by Ordnance Survey (via the

19 Digimap Licence). The photogrammetric Digital Surface Model was made available by

20 Ordnance Survey.

\section{References}

22 Aksoezen, M., Daniel, M., Hassler, U., and Kohler, N., 2015. Building age as an 23 indicator for energy consumption. Energy and Buildings, 87, 74-86. 
1 Alexander, D.K., Lannon, S., and Linovski, O., 2009. the Identification and Analysis of

Barnsley, M.J. and Barr, S.L., 1997. Distinguishing urban land-use categories in fine spatial resolution land-cover data using a graph-based, structural pattern recognition system. Computers, Environment and Urban Systems, 21 (3-4), 209225.

Beck, A., Long, G., Boyd, D.S., Rosser, J.F., Morley, J., Duffield, R., Sanderson, M., and Robinson, D., 2018. Automated classification metrics for energy modelling of residential buildings in the UK with open algorithms. Environment and Planning B: Urban Analytics and City Science.

BEIS, 2017. Energy Consumption in the Uk. Department for Business, Energy and Industrial Strategy, (July).

Biljecki, F., Ledoux, H., and Stoter, J., 2017. Generating 3D city models without elevation data. Computers, Environment and Urban Systems, Under review, 64, 118.

Biljecki, F. and Sindram, M., 2017. Estimating building age with 3D GIS. ISPRS Ann. Photogramm. Remote Sens. Spatial Inf. Sci., IV-4/W5 (October), 17-24.

Bishop, C.M., 1995. Neural networks for pattern recognition. Oxford University Press.

Boykov, Y. and Kolmogorov, V., 2004. An Experimental Comparison of Min-Cut/MaxFlow Algorithms for Energy Minimization in Vision. IEEE transactions on Pattern Analysis and Machine Intelligence, 26 (9), 1124-1137.

Boykov, Y., Veksler, O., and Zabih, R., 2001. Efficient Approximate Energy Minimization via Graph Cuts. IEEE transactions on Pattern Analysis and Machine Intelligence, 20 (12), 1222-1239.

Breiman, L., 1984. Classification and Regression Trees. New York: Routeledge.

Breiman, L., 2001. Random forests. Machine Learning, 45 (1), 5-32. 
1 Brown, F. and Steadman, J., 1991. The morphology of British housing: an empirical basis for policy and research. Part I: Functional and dimensional characteristics. Environment and Planning B: Planning and Design, 18, 277-299.

Davies, M. and Oreszczyn, T., 2012. The unintended consequences of decarbonising the built environment: A UK case study. Energy and Buildings, 46, 80-85.

Davila, C.C., Reinhart, C., and Bemis, J., 2016. Modeling Boston: A workflow for the generation of complete urban building energy demand models from existing urban geospatial datasets. Energy, 117, 237-250.

DCLG, 2015. Deparment for Communities and Local Government, English Housing Survey: Housing Stock Report, 2014-2015.

Fan, H., Zipf, A., Fu, Q., and Neis, P., 2014. Quality assessment for building footprints data on OpenStreetMap. International Journal of Geographical Information Science, 28 (4), 700-719.

Firth, S.K., Lomas, K.J., and Wright, A.J., 2010. Targeting household energy-efficiency measures using sensitivity analysis. Building Research and Information, 38 (1), $24-41$.

Fulkerson, B., Vedaldi, A., and Soatto, S., 2009. Class Segmentation and Object Localization with Superpixel Neighborhoods. In: Proceedings of the International Conference on Computer Vision.

Hamilton, I.G., Steadman, P.J., Bruhns, H., Summerfield, A.J., and Lowe, R., 2013. Energy efficiency in the British housing stock: Energy demand and the Homes Energy Efficiency Database. Energy Policy, 60, 462-480.

Hastie, T., Tibshirani, R., and Friedman, J., 2009. The Elements of Statistical Learning: Data Mining, Inference, and Prediction. New York, NY: Springer New York.

Hermosilla, T., Palomar-Vázquez, J., Balaguer-Beser, Á., Balsa-Barreiro, J., and Ruiz, L.A., 2014. Using street based metrics to characterize urban typologies. Computers, Environment and Urban Systems, 44, 68-79.

Hermosilla, T., Ruiz, L.A., Recio, J.A., and Cambra-López, M., 2012. Assessing 
contextual descriptive features for plot-based classification of urban areas. Landscape and Urban Planning, 106 (1), 124-137.

Hernandez-Vela, A., Zlateva, N., Marinov, A., Reyes, M., Radeva, P., Dimov, D., and Escalera, S., 2012. Graph cuts optimization for multi-limb human segmentation in depth maps. Proceedings of the IEEE Computer Society Conference on Computer Vision and Pattern Recognition, 726-732.

Van Hoesen, J. and Letendre, S., 2013. Characterizing the spatiotemporal evolution of building-stock age in Poultney, Vermont: A GIS-based approach to improve thermal efficiency in historical buildings. Environment and Planning B: Planning and Design, 40 (4), 630-643.

Huang, H., Kieler, B., and Sester, M., 2013. Urban building usage labeling by geometric and context analyses of the footprint data. In: Proceeding of 26th international cartographic conference (ICC).

James, G., Witen, D., Hastie, T., and Tibshirani, R., 2007. An Introduction to Statistical Learning with Applications in R. Performance Evaluation.

Jensen, F. V, 1996. Introduction to Bayesian Networks. 1st ed. Secaucus, NJ, USA: Springer-Verlag New York, Inc.

Kolmogorov, V. and Zabih, R., 2004. What Energy Functions can be Minimized via Graph Cuts? IEEE transactions on Pattern Analysis and Machine Intelligence, 26 (2), 147-159.

Landis, J.R. and Koch, G.G., 1977. The Measurement of Observer Agreement for Categorical Data. Biometrics, 33 (1), 159-174.

Long, G., Alwany, M., and Robinson, D., 2015. Building typologies simulation report, Nottingham. [online]. Available from: http://www.insmartenergy.com/wpcontent/uploads/2014/12/D.2.1.-Simulation_Report_for_building_TypologiesNottingham.pdf [Accessed 12 Jul 2017].

Lucon, O., Ürge-Vorsatz, D., Zain Ahmed, A., Akbari, H., Bertoldi, P., Cabeza, L.F., Eyre, N., Gadgil, A., Harvey, L.D.D., Jiang, Y., Liphoto, E., Mirasgedis, S., 
Murakami, S., Parikh, J., Pyke, C., and Vilariño, M.V., 2014. Buildings. Climate Change 2014: Mitigation of Climate Change. Contribution of Working Group III to the Fifth Assessment Report of the Intergovernmental Panel on Climate Change, $33,1-66$.

Mavrogianni, A., Wilkinson, P., Davies, M., Biddulph, P., and Oikonomou, E., 2012. Building characteristics as determinants of propensity to high indoor summer temperatures in London dwellings. Building and Environment, 55, 117-130.

Meinel, G., Hecht, R., and Herold, H., 2009. Analyzing building stock using topographic maps and GIS. Building Research and Information, 37 (5-6), 468482.

Nouvel, R., Zirak, M., Coors, V., and Eicker, U., 2017. The influence of data quality on urban heating demand modeling using 3D city models. Computers, Environment and Urban Systems, 64, 68-80.

ONS Geography, 2011. Output Areas (December 2011) Boundaries [online]. Available from: http://geoportal.statistics.gov.uk/datasets/output-area-december-2011-fullextent-boundaries-in-england-and-wales [Accessed 6 Sep 2017].

Ordnance Survey, 2016. AddressBase Plus [online]. Available from: https://www.ordnancesurvey.co.uk/business-andgovernment/products/addressbase-plus.html [Accessed 9 Dec 2016].

Ordnance Survey, 2017. OS MasterMap Topography Layer [online]. Available from: https://www.ordnancesurvey.co.uk/docs/technical-specifications/os-mastermaptopography-layer-technical-specification.pdf [Accessed 4 Jan 2018].

Oshiro, T.M. and Perez, P.S., 2012. Machine Learning and Data Mining in Pattern Recognition, 7376 (July 2012).

RIBA, 2015. Space Standards For Homes. Royal Institute of British Architects (RIBA) technical report. London.

Schindler, K., 2012. An overview and comparison of smooth labeling methods for landcover classification. IEEE Transactions on Geoscience and Remote Sensing, 50 
2 Shorrock, L.D. and Dunster, J.E., 1997. The physically-based model BREHOMES and

Shorrock, L.D., Henderson, J., and Utley, J.I., 2005. Reducing carbon emissions from the UK housing stock. BRE Press., 80.

Sousa, G., Jones, B.M., Mirzaei, P.A., and Robinson, D., 2017. A review and critique of UK housing stock energy models, modelling approaches and data sources. Energy and Buildings, 151, 66-80.

Steimen, S., Fäh, D., Giardini, D., Bertogg, M., and Tschudi, S., 2004. Reliability of building inventories in seismic prone regions. Bulletin of Earthquake Engineering, $2(3), 361-388$.

Strobl, C., Boulesteix, A.L., Zeileis, A., and Hothorn, T., 2007. Bias in random forest variable importance measures: Illustrations, sources and a solution. $B M C$ Bioinformatics, 8.

Tooke, T.R., Coops, N.C., and Webster, J., 2014. Predicting building ages from LiDAR data with random forests for building energy modeling. Energy and Buildings, 68 (PARTA), 603-610.

Uzielli, M., Catani, F., Tofani, V., and Casagli, N., 2015. Risk analysis for the Ancona landslide-II: estimation of risk to buildings. Landslides, 12 (1), 83-100.

Wang, Y., Zhang, L., Takis Mathiopoulos, P., and Deng, H., 2015. A Gestalt rules and graph-cut-based simplification framework forurban building models. International Journal of Applied Earth Observation and Geoinformation, 35 (PB), 247-258.

Wieland, M., Pittore, M., Parolai, S., Zschau, J., Moldobekov, B., and Begaliev, U., 2012. Estimating building inventory for rapid seismic vulnerability assessment: Towards an integrated approach based on multi-source imaging. Soil Dynamics and Earthquake Engineering, 36, 70-83.

Wilson, A. and Boehland, J., 2005. Small is Beautiful U.S. House Size, Resource Use, 

and the Environment. Journal of Industrial Ecology, 9 (1-2), 277-287.

2 Wurm, M., Schmitt, A., and Taubenböck, H., 2016. Building Types' Classification 3 Using Shape-Based Features and Linear Discriminant Functions. IEEE Journal of $4 \quad$ Selected Topics in Applied Earth Observations and Remote Sensing, 9 (5), 190151912.

6 Yu, B., Liu, H., Wu, J., Hu, Y., and Zhang, L., 2010. Automated derivation of urban 7 building density information using airborne LiDAR data and object-based method. $8 \quad$ Landscape and Urban Planning, 98 (3-4), 210-219.

9 Zaharia, M., Chowdhury, M., Franklin, M.J., Shenker, S., and Stoica, I., 2010. Spark: 10 Cluster Computing with Working Sets. HotCloud'10 Proceedings of the 2nd 11 USENIX conference on Hot topics in Cloud Computing, 10. 